\title{
Early postoperative changes in cerebral oxygen metabolism following neonatal cardiac surgery: Effects of surgical duration
}

\author{
Erin M. Buckley, PhD, ${ }^{\mathrm{a}, \mathrm{b}}$ Jennifer M. Lynch, MS, ${ }^{\mathrm{b}}$ Donna A. Goff, MD, ${ }^{\mathrm{c}, \mathrm{d}}$ Peter J. Schwab, BA, ${ }^{\mathrm{a}}$ \\ Wesley B. Baker, MS, ${ }^{\mathrm{b}}$ Turgut Durduran, PhD, ${ }^{\mathrm{e}}$ David R. Busch, PhD, ${ }^{\mathrm{b}}$ Susan C. Nicolson, MD, ${ }^{\mathrm{f}}$ \\ Lisa M. Montenegro, MD, ${ }^{\mathrm{f}}$ Maryam Y. Naim, MD, ${ }^{\mathrm{g}}$ Rui Xiao, PhD,${ }^{\mathrm{h}}$ Thomas L. Spray, MD, ${ }^{\mathrm{i}}$ \\ A. G. Yodh, PhD, ${ }^{\mathrm{b}}$ J. William Gaynor, MD, ${ }^{\mathrm{i}}$ and Daniel J. Licht, $\mathrm{MD}^{\mathrm{a}}$
}

\begin{abstract}
Objective: The early postoperative period following neonatal cardiac surgery is a time of increased risk for brain injury, yet the mechanisms underlying this risk are unknown. To understand these risks more completely, we quantified changes in postoperative cerebral metabolic rate of oxygen $\left(\mathrm{CMRO}_{2}\right)$, oxygen extraction fraction $(\mathrm{OEF})$, and cerebral blood flow (CBF) compared with preoperative levels by using noninvasive optical modalities.
\end{abstract}

\begin{abstract}
Methods: Diffuse optical spectroscopy and diffuse correlation spectroscopy were used concurrently to derive cerebral blood flow and oxygen utilization postoperatively for 12 hours. Relative changes in $\mathrm{CMRO}_{2}, \mathrm{OEF}$, and $\mathrm{CBF}$ were quantified with reference to preoperative data. A mixed-effect model was used to investigate the influence of total support time and deep hypothermic circulatory arrest duration on relative changes in $\mathrm{CMRO}_{2}, \mathrm{OEF}$, and CBF.

Results: Relative changes in $\mathrm{CMRO}_{2}$, OEF, and CBF were assessed in 36 patients, 21 with single-ventricle defects and 15 with 2-ventricle defects. Among patients with single-ventricle lesions, deep hypothermic circulatory arrest duration did not affect relative changes in $\mathrm{CMRO}_{2}$, CBF, or OEF $(P>.05)$. Among 2-ventricle patients, total support time was not a significant predictor of relative changes in $\mathrm{CMRO}_{2}$ or $\mathrm{CBF}(P>.05)$, although longer total support time was associated significantly with greater increases in relative change of postoperative $\mathrm{OEF}(P=.008)$.
\end{abstract}

Conclusions: Noninvasive diffuse optical techniques were used to quantify postoperative relative changes in $\mathrm{CMRO}_{2}, \mathrm{CBF}$, and $\mathrm{OEF}$ for the first time in this observational pilot study. Pilot data suggest that surgical duration does not account for observed variability in the relative change in $\mathrm{CMRO}_{2}$, and that more comprehensive clinical studies using the new technology are feasible and warranted to elucidate these issues further. (J Thorac Cardiovasc Surg 2013;145:196-205)

Supplemental material is available online.

\footnotetext{
From the Divisions of Neurology, ${ }^{\mathrm{a}}$ Cardiology, ${ }^{\mathrm{c}}$ Cardiothoracic Anesthesia, ${ }^{\mathrm{f}}$ Critical Care Medicine, ${ }^{\mathrm{g}}$ and Cardiothoracic Surgery, ${ }^{\mathrm{i}}$ The Children's Hospital of Philadelphia, Philadelphia, Pa; the Departments of Physics and Astronomy ${ }^{\mathrm{b}}$ and Biostatistics and Epidemiology, ${ }^{\mathrm{h}}$ University of Pennsylvania, Philadelphia, $\mathrm{Pa}$; the Division of Pediatric Cardiology, ${ }^{\mathrm{d}}$ Loma Linda University Children's Hospital, Loma Linda, Calif; and the ICFO-Institut de Ciències Fotòniques, ${ }^{\mathrm{e}}$ Barcelona, Spain.

This work was supported by the National Institutes of Health (HNS072338: principle investigator [PI], Dr Licht; NS-052380: PI, Dr Licht; NS-060653: PI, Dr Yodh; P41-EB015893: Dr Yodh; T32NS007413: Dr Buckley), by the Dana Foundation (PI, Dr Licht), by Fundacio Cellex Barcelona (PI, Dr Durduran), and by the Steve and Judy Wolfson Family Trust (PI, Dr Licht).

Disclosures: Authors have nothing to disclose with regard to commercial support.

Read at the 92nd Annual Meeting of The American Association for Thoracic Surgery, San Francisco, California, April 28-May 2, 2012.

Received for publication May 3, 2012; revisions received Aug 21, 2012; accepted for publication Sept 21, 2012; available ahead of print Oct 29, 2012.

Address for reprints: Erin M. Buckley, PhD, 3231 Walnut St, Philadelphia, PA 19104 (E-mail: buckley@nmr.mgh.harvard.edu).

0022-5223/\$36.00

Copyright (c) 2013 by The American Association for Thoracic Surgery

http://dx.doi.org/10.1016/j.jtcvs.2012.09.057
}

The past 3 decades have seen a dramatic increase in survival rates of infants born with congenital heart defects (CHDs) following surgical intervention. As a result, clinical focus has shifted toward the short- and long-term neurologic sequelae that often accompany otherwise successful cardiac repairs. ${ }^{1}$ Within 2 weeks after surgery, magnetic resonance imaging studies have revealed that these infants have a remarkably high $(>50 \%)$ incidence of hypoxic-ischemic white matter injury in the form of periventricular leukomalacia, ${ }^{1-3}$ an elevated risk of stroke, ${ }^{4}$ and brain immaturity. ${ }^{2}$ By school age, children born with various forms of CHDs often exhibit problems with academic achievement, fine and gross motor function, visual-spatial skills, and executive function. ${ }^{1,5-8}$

The mechanisms underlying this increased perioperative risk of brain injury and subsequent neurocognitive impairments are not fully understood. However, evidence suggests links between these impairments and increased cerebral metabolic demands or decreased cerebral oxygen delivery secondary to cardiopulmonary bypass (CPB) and/or deep hypothermic circulatory arrest (DHCA), compromised 


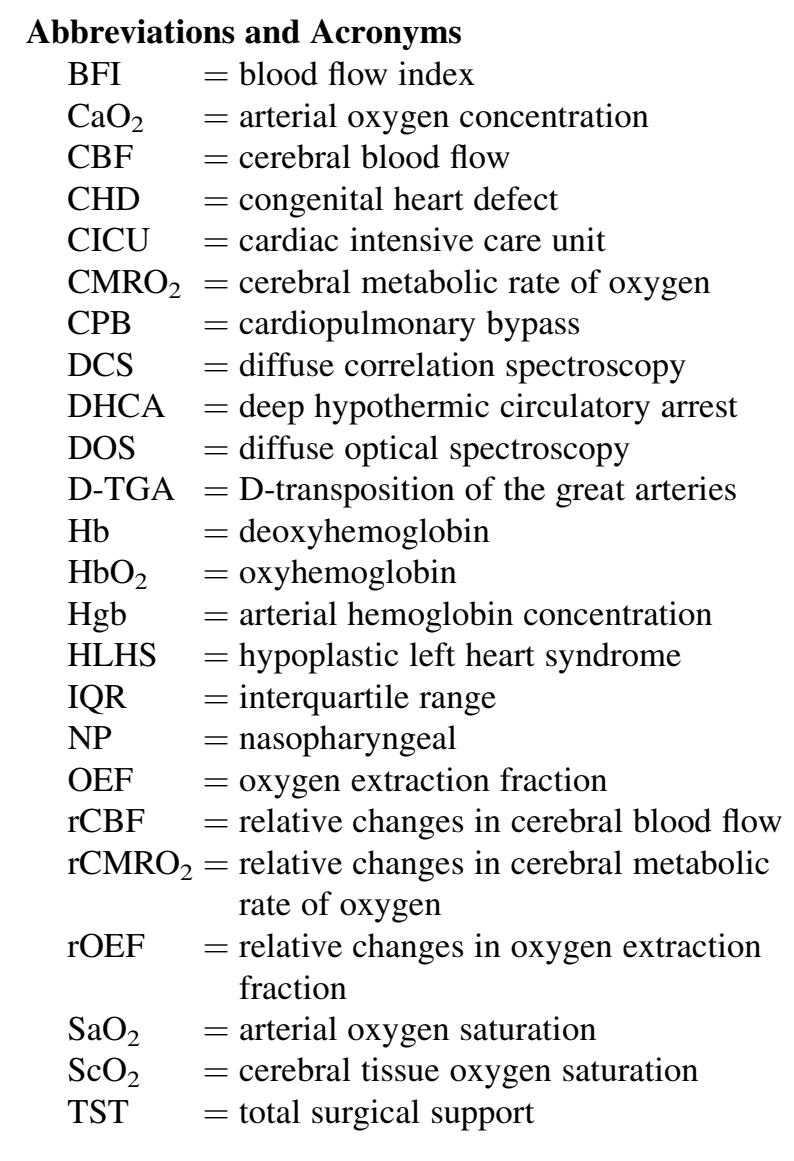

cardiorespiratory function with hemodynamic instability and hypoxemia, loss of cerebral autoregulation, and/or the systemic inflammatory response to surgery. ${ }^{2,9}$ Several reports have shown that longer duration of $\mathrm{CPB}$ and/or DHCA may be linked with impaired neurocognitive outcome $^{3,6}$; however, other research has failed to find similar links. $2,4,7,8$

Despite numerous studies suggesting a link between increased postoperative metabolic demands and subsequent cerebral injury, quantification of cerebral metabolic rate of oxygen $\left(\mathrm{CMRO}_{2}\right)$ in neonates with CHDs is limited, in large part because of technologic and logistic challenges of these measurements. Pioneering work by Greeley and colleagues ${ }^{10-13}$ demonstrated that, after surgery, $\mathrm{CMRO}_{2}$ is suppressed in patients undergoing DHCA but not in patients exposed to moderate hypothermia, nor in patients exposed to deep hypothermia with continuous flow. However, the measurement of $\mathrm{CMRO}_{2}$ after return from the operating room during the early postoperative period has not yet been reported, nor has any connection between postoperative $\mathrm{CMRO}_{2}$ and the support techniques used during surgery.
To address these clinical questions, we used diffuse optical spectroscopy (DOS) and diffuse correlation spectroscopy (DCS) to monitor early postoperative changes in $\mathrm{CMRO}_{2}$ compared with preoperative levels in neonates with CHD with both single- and 2-ventricle cardiac lesions, respectively. Furthermore, we investigated the potential influence of the duration of total surgical support time (TST) and/or DHCA on postoperative cerebral hemodynamics resulting from changes in metabolic demands.

\section{METHODS \\ Patient Population}

This research is a prospective observational study. Inclusion/exclusion criteria are described in Licht and colleagues. ${ }^{14}$ In brief, all otherwise healthy full-term (ie, gestational age $>37$ weeks) newborn infants with complex CHDs admitted for surgical intervention with $\mathrm{CPB}$ were recruited with institutional review board approval.

\section{Surgical Strategies}

Operations were performed by 3 cardiac surgeons and a team of cardiac anesthesiologists. pH-stat blood gas management was used. In general, during normothermic or mild hypothermic CPB (nasopharyngeal $[\mathrm{NP}]$ temperature $>28^{\circ} \mathrm{C}$ ), the pump flow rate was maintained at 100 to $150 \mathrm{~mL} / \mathrm{kg} /$ minute to achieve a mean arterial pressure of 30 to $55 \mathrm{~mm} \mathrm{Hg}$. For deep hypothermic continuous CPB (NP temperature $<22^{\circ} \mathrm{C}$ ), a pump flow rate of 25 to $50 \mathrm{~mL} / \mathrm{kg} /$ minute was used. Note, these general guidelines were modified as needed according to the clinical situation. Deep hypothermic circulatory arrest was used selectively at the surgeon's discretion, not according to a predetermined protocol. Before DHCA, patients underwent core and surface cooling with topical hypothermia of the head to an NP temperature of $18^{\circ} \mathrm{C}$. Deep hypothermic circulatory arrest was used for all patients undergoing stage 1 reconstruction for hypoplastic left heart syndrome (HLHS) or HLHS variants. No patient received continuous antegrade cerebral perfusion during the repair. The arterial switch operation and other repairs of patients with 2-ventricle CHDs were performed with continuous CPB. Modified ultrafiltration was performed in all patients after CPB. Cerebral oximetry was not used to guide intraoperative management.

\section{Postoperative Management}

Patients were monitored postoperatively in a dedicated cardiac intensive care unit (CICU) with continuous electrocardiography, pulse oximetry, invasive arterial blood pressure transduction by radial or ulnar catheterization, and right atrial pressure transduction by transthoracic right atrial catheters, as well as intermittent arterial blood gas analysis and bedside echocardiography as needed (ie, for hemodynamic instability or desaturation). Arterial blood gas analyses were performed as part of clinical care.

\section{Cerebral Monitoring}

Diffuse optical spectroscopy and diffuse correlation spectroscopy (DOS and DCS, respectively) use near-infrared light to probe static and dynamic optical properties of cortical brain tissue noninvasively. The custom-made optical instrument used in this study combined frequency-domain DOS with DCS on a mobile cart that is ideal for bedside monitoring. ${ }^{15}$

Frequency-domain DOS (also known as near infrared spectroscopy) is a widely accepted modality for measurement of cerebral oxy- and deoxyhemoglobin concentration ([ $\left.\mathrm{HbO}_{2}\right]$ and $[\mathrm{Hb}]$, respectively). Frequency-domain DOS uses photon diffusion theory to relate the measured amplitude 
attenuation and phase shift of modulated, multiply scattered light detected on the tissue surface to the wavelength-dependent absorption $\left(\mu_{a}\right)$ and scattering $\left(\mu_{s}^{\prime}\right)$ properties of the probed tissue. ${ }^{16}$ Our DOS system (Imagent; ISS, Inc, Champaign, Ill) used 8 laser diode sources modulated at 110 $\mathrm{MHz}$ and operating at 2 wavelengths $(688 \mathrm{~nm}$ and $826 \mathrm{~nm})$, and a photomultiplier tube detector with gain modulation of $110 \mathrm{MHz}+5 \mathrm{kHz}$ for heterodyne detection at $5 \mathrm{kHz}$.

Diffuse correlation spectroscopy is a relatively new technology that has been used to monitor cerebral blood flow (CBF) noninvasively. Diffuse correlation spectroscopy monitors temporal fluctuations in the reflected light intensity measured at points on the tissue surface. These temporal fluctuations are caused primarily by photons that have been scattered by moving red blood cells. ${ }^{16-18}$ Specifically, the temporal intensity autocorrelation function of detected light is computed, and correlation diffusion theory ${ }^{16}$ is used to extract a tissue blood flow index (BFI, measured in square centimeters per second). Previous studies in clinical and animal model systems have shown that changes in BFI relative to a baseline are in agreement with changes in $\mathrm{CBF}$ relative to a baseline measured by other gold standard $\mathrm{CBF}$ techniques. ${ }^{15,16,19}$ The DCS instrument used in this work has been described in detail elsewhere. ${ }^{15}$

The patient interface for this hybrid DOS/DCS instrument consisted of a custom-made flexible black rubber probe (ISS, Inc) that was held manually against the patient's forehead. This probe houses fiber optics for both DOS and DCS. For DOS, we used 4 source-detector separations (1.5, 2.0, 2.5 , and $3.0 \mathrm{~cm}$ ), with 2 fibers (ie, one for each wavelength at each separation) and 1 detection fiber. For DCS, 8 single-mode detection fibers were bundled and placed $2.5 \mathrm{~cm}$ away from the source fiber (on the tissue surface).

\section{Study Protocol}

Diffuse optical and diffuse correlation spectroscopy measurements of cerebral tissue oxygen saturation $\left(\mathrm{ScO}_{2}\right.$, measured as a percentage $)$ and BFI were made multiple times on the day of surgery, both pre- and postoperatively. The measurement protocol and data analysis are described in detail in Appendix 1. We defined $t=0$ to be the time at which the patient was taken off CPB support. Each patient had 1 measurement immediately prior to surgery (typically around $t=-4$ hours) and 1 measurement immediately after patient return to the CICU after surgery (typically at $t=1.5$ hours); multiple follow-up measurements were taken every 2 to 3 hours for the first 12 hours after surgery. During these brief measurement sessions, no ventilator changes were made during a session, blood loss was negligible, and vital signs were stable.

Arterial oxygen saturation $\left(\mathrm{SaO}_{2}\right.$, measured as a percentage $)$ and hemoglobin concentration (Hgb, measured in grams per deciliter) were calculated from clinically indicated arterial blood gases. These values were used to compute oxygen extraction fraction (OEF), arterial oxygen concentration $\left(\mathrm{CaO}_{2} \equiv 0.0134 \times \mathrm{SaO}_{2} \times \mathrm{Hgb}\right.$; measured in milliliters per deciliter), and $\mathrm{CMRO}_{2}$. Last, relative changes in postoperative $\mathrm{OEF}, \mathrm{CBF}$, and $\mathrm{CMRO}_{2}$ (ie, rOEF, rCBF, and $\mathrm{rCMRO}_{2}$ ) from baseline (preoperative) levels were computed so that each patient served as his or her own control (see Appendix 1).

\section{Statistical Analysis}

For analysis purposes, patients were grouped according to cardiac defect (ie, 1-ventricle vs 2-ventricle defects). Group 1 consisted of patients with single-ventricle anatomy who received first-stage surgical palliation of HLHS and HLHS variants. Group 2 consisted of patients with 2-ventricle anatomy with D-transposition of the great arteries (D-TGA). Intraoperative TST was defined as CPB plus DHCA duration. To test for differences in patient characteristics between groups, we carried out a Wilcoxon rank-sum test for continuous variables. To investigate differences in postoperative systemic and cerebral hemodynamics on return to the CICU from preoperative levels, we used a Wilcoxon matched-pair test (for absolute measures such as $\mathrm{SaO}_{2}, \mathrm{Hgb}$, etc) and a Wilcoxon signed-rank test (for relative measures; ie, rCBF, $\mathrm{rOEF}$, and $\mathrm{rCMRO}_{2}$ ).

For patients in group 1, the effect of DHCA duration on the time profiles of $\mathrm{rCMRO}_{2}, \mathrm{rCaO}_{2}, \mathrm{rCBF}$, and $\mathrm{rOEF}$ was examined using a linear mixed-effect model with subject-specific random effects to account for within-subject correlations resulting from repeated measures. The model was used to predict the mean outcome variable (ie, $\mathrm{rCMRO}_{2}, \mathrm{rCBF}$, $\mathrm{rCaO}_{2}$, or rOEF) as a function of DHCA duration and time after removal from CPB. Logarithmic transformation was applied to all outcome variables to make the data more normally distributed. A random slope and intercept model was used for between-subject variation. Compound symmetry covariance structure, which assumed that correlations between all pairs of measures within the same subject are the same, was used in the mixed-effect model. A similar mixed-effect model was used in group 2 to predict the mean outcome variable as a function of TST and as a function of time after removal from CPB. All analyses were performed using SAS 9.3 statistical software (SAS Institute Inc, Cary, NC). Statistical significance was declared for $P$ values $<.05$.

\section{RESULTS}

Between March 2010 and July 2012, 39 neonates were recruited for study of pre- and postoperative cerebral hemodynamics. One patient was excluded from the study because he returned on extracorporeal membrane oxygenation; 2 patients were excluded because of poor pre- or postoperative optical data quality. Thus, the remaining 36 patients were included in our analysis. Group 1 consisted of 21 patients with HLHS patients; group 2 consisted of 15 patients with D-TGA. Of the 21 patients in group 1, 4 had aortic atresia/ mitral atresia $(19 \%, 1$ with intact atrial septum), 8 had aortic atresia/mitral stenosis (38\%, 1 with intact atrial septum), 6 had aortic stenosis/mitral stenosis (29\%), 1 had a severely unbalanced atrioventricular canal with arch hypoplasia $(5 \%)$, and 2 had double-outlet right ventricle with aortic atresia/mitral stenosis $(10 \%, 1$ with a ventricular septal defect). Nine of 15 patients with D-TGA (60\%) had balloon atrial septostomy performed 2 to 4 days prior to surgery.

Patient demographics are summarized in Table 1. Patients were born full term and went to surgery within the first week of life. No differences in gestational age, birth weight, day of life at surgery, or head circumference were observed between groups. Median (interquartile range [IQR]) surgical parameters, including CPB hematocrit, temperature nadir, cooing time, TST, and DHCA duration, are given in Table 2. The median intraoperative TST for the entire cohort was 87 minutes (IQR, 70-116 minutes). No difference in TST was observed between groups $(P=.62)$.

Preoperative systemic and cerebral parameters separated by cardiac lesion are summarized in Table 3 (see also Appendix E1). Patients with single-ventricle CHDs had significantly higher $\mathrm{SaO}_{2}$ and $\mathrm{CaO}_{2}$ levels than those with 2 -ventricle lesions $(P<.001$ and $P=.01$, respectively). No differences between groups were observed in preoperative arterial $\mathrm{Hgb}, \mathrm{ScO}_{2}$, or OEF. The median (IQR) of the first postoperative value of each of these systemic and 
TABLE 1. Summary of median (interquartile range) of demographic patient characteristics

\begin{tabular}{lccc}
\hline \multicolumn{1}{c}{ Variable } & Group 1 & Group 2 & $\boldsymbol{P}$ value \\
\hline Gender, male:female; $\mathrm{n}$ & $12: 9$ & $8: 7$ & - \\
Gestation age, weeks & $38.7(38.0-39.4)$ & $38.9(38.0-39.1)$ & .97 \\
Birth weight, kg & $3.2(3.0-3.5)$ & $3.4(3.2-3.8)$ & .09 \\
Day of life & $3(2-5)$ & $3(3-4)$ & .91 \\
Head circumference, cm & $33.6(33.0-34.5)$ & $34.1(33.5-35.0)$ & .13 \\
\hline
\end{tabular}

A Wilcoxon rank-sum test was used to test for significant differences between groups.

cerebral measures is also displayed in Table 3 (see also Appendix E1). These data were obtained during normothermia and relative hemodynamic stability after the patient returned to the CICU at a median time of 1.5 hours after removal from CPB. For patients with single-ventricle lesions, $\mathrm{SaO}_{2}, \mathrm{CaO}_{2}$, and $\mathrm{ScO}_{2}$ decreased significantly from preoperative values after surgery $(P<.001, P=.006$, $P=.001$, respectively). In addition, $\mathrm{rCBF}$ and $\mathrm{rCMRO}_{2}$ decreased after surgery $(P=.003$ and $P=.03)$; in other words, the medians for $\mathrm{rCBF}$ and $\mathrm{rCMRO}_{2}$ were both less than $100 \%$ of preoperative values. For patients with 2-ventricle lesions, $\mathrm{SaO}_{2}, \mathrm{CaO}_{2}$, and rOEF increased significantly after surgery $(P<.001, P=.002, P=.005$, respectively); $\mathrm{rCBF}$ decreased $(P=.01)$, and $\mathrm{rCMRO}_{2}$ did not change $(P=.17)$ on average from preoperative levels.

Figures 1 and 2 show the relative changes in all outcome variables (ie, $\mathrm{rCMRO}_{2}, \mathrm{rCBF}$, $\mathrm{OEF}$, and $\mathrm{rCaO}_{2}$ ) from preoperative levels for patients with single- and 2-ventricle lesions, respectively. In both groups, the observed cerebral hemodynamic changes after surgery were quite heterogeneous across individual patients. However, these figures do suggest trends in the population average hemodynamic responses to surgery, such as in Figure $1, D$, in which $\mathrm{rCaO}_{2}$ for single-ventricle patients appears to increase from its preoperative level, which is in agreement with the statistical test in Table 4.

Among patients with single-ventricle heart lesions, we performed a mixed-effect model to assess the effects of DHCA duration on our outcome variables using DHCA duration and time after removal from $\mathrm{CPB}$ as model predictors. The results of this analysis are summarized in Table 4. Deep hypothermic circulatory arrest duration did not have a significant effect on changes in $\mathrm{rCMRO}_{2}$

TABLE 2. Summary of median (interquartile range) of surgical parameters

\begin{tabular}{lccc}
\hline \multicolumn{1}{c}{ Variable } & Group 1 & Group 2 & $\boldsymbol{P}$ value \\
\hline Hematocrit, $\%$ & $30(29-34)$ & $31(29-33)$ & .66 \\
Temperature nadir, ${ }^{\circ} \mathrm{C}$ & $17.6(17.1-18.8)$ & $33.7(31.4-35.2)$ & $<.0001$ \\
Cooling time, min & $17(15-21)$ & - & - \\
Total support time, min & $86(74-102)$ & $111(53-138)$ & .62 \\
DHCA duration, min & $44(41-54)$ & - & - \\
\hline
\end{tabular}

A Wilcoxon rank-sum test was used to test for significant differences between groups. $D H C A$, Deep hypothermic circulatory arrest.
TABLE 3. Summary of median (interquartile range) pre- and first postoperative systemic and cerebral hemodynamic variables measured

\begin{tabular}{clccc} 
CHD type & \multicolumn{1}{c}{ Variable } & Preoperative & Postoperative & $\begin{array}{c}\boldsymbol{P} \\
\text { value }\end{array}$ \\
\hline One ventricle & $\mathrm{SaO}_{2}, \%$ & $88(85-90)$ & $74(66-77)$ & $<.001$ \\
& $\mathrm{Hgb}, \mathrm{g} / \mathrm{dL}$ & $13.9(13.3-14.6)$ & $15.0(14.0-15.7)$ & .044 \\
& $\mathrm{CaO}, \mathrm{mL} / \mathrm{dL}$ & $16.2(15.3-17.3)$ & $14.4(12.9-15.8)$ & .006 \\
& $\mathrm{ScO}_{2}, \%$ & $52(43-59)$ & $42(38-46)$ & .001 \\
& $\mathrm{rOEF}, \%$ & $100^{*}$ & $112(76-162)$ & .19 \\
& $\mathrm{rCBF}, \%$ & $100^{*}$ & $72(39-100)$ & .003 \\
& $\mathrm{rCMRO}_{2}, \%$ & $100^{*}$ & $73(33-96)$ & .028 \\
Two ventricles & $\mathrm{SaO}_{2}, \%$ & $78(67-81)$ & $99(95-100)$ & $<.001$ \\
& $\mathrm{Hgb}^{2}, \mathrm{~g} / \mathrm{dL}$ & $13.6(12.3-15.2)$ & $13.9(12.3-15.2)$ & .75 \\
& $\mathrm{CaO}_{2}, \mathrm{~mL} / \mathrm{dL}$ & $13.2(12.1-15.7)$ & $18.3(15.9-20.4)$ & .002 \\
& $\mathrm{ScO}, \%$ & $54(48-55)$ & $56(45-62)$ & .80 \\
& $\mathrm{rOEF}_{2}, \%$ & $100^{*}$ & $145(107-222)$ & .005 \\
& $\mathrm{rCBF}, \%^{2} \%$ & $100^{*}$ & $70(40-90)$ & .013 \\
& $\mathrm{rCMRO}_{2}, \%$ & $100^{*}$ & $121(59-207)$ & .17 \\
\hline
\end{tabular}

A Wilcoxon matched-pair test was used for differences in $\mathrm{SaO}_{2}, \mathrm{Hgb}, \mathrm{CaO}_{2}$, and $\mathrm{ScO}_{2}$ from before the operation to after the operation. A Wilcoxon signed-rank test was used to test for changes in $\mathrm{rOEF}, \mathrm{rCBF}$, and $\mathrm{rCMRO}_{2}$ from preoperative levels. $\mathrm{CHD}$, Congenital heart defect; $\mathrm{SaO}_{2}$, arterial oxygen saturation; $\mathrm{Hgb}$, hemoglobin; $\mathrm{ScO}_{2}$, cerebral tissue oxygen saturation; $\mathrm{CaO}_{2}$, arterial oxygen concentration, $r O E F$, relative change in postoperative oxygen extraction fraction; $r C B F$, relative change in postoperative cerebral blood flow; $r \mathrm{CMRO}_{2}$, relative change in postoperative cerebral metabolic rate of oxygen. *Each patient started at $100 \%$ and thus served as his or her own control.

$(P=.45), \operatorname{rOEF}(P=.96)$, or $\mathrm{rCBF}(P=.87)$ compared with preoperative levels. However, longer DHCA durations were associated significantly with a greater increase in postoperative changes in $\mathrm{rCaO}_{2}$ from preoperative levels $(P=.0001)$. In addition, of the 4 outcome variables, only $\mathrm{rCaO}_{2}$ showed a significant increase with time after removal from CPB $(P<.0001)$. Among several possible explanations, this increase in $\mathrm{rCaO}_{2}$ with time may reflect the degree of pulmonary inflammation or injury from prolonged DHCA, with later recovery.

For patients with 2-ventricle CHDs, we used a mixedeffects model to assess the effects of TST on our outcome variables using TST and time after removal from CPB as model predictors. The results of this analysis are summarized in Table 4. Although longer TST is associated with a greater increase in the change in $\operatorname{rOEF}(P=.008)$, TST did not affect changes significantly in $\mathrm{rCMRO}_{2}(P=.29)$, $\mathrm{CBF}(P=.47)$, or $\mathrm{CaO}_{2}(P=.73)$. In addition, of the 4 outcome variables, only rCBF showed a significant increase over time after removal from CPB $(P=.027)$.

\section{DISCUSSION}

This work describes trends in optically measured $\mathrm{rCMRO}_{2}$, rOEF, and rCBF after return to the CICU after neonatal cardiac surgery (ie, during the first 12 hours after removal from bypass) for 2 distinct patient groups-namely, those with single- and 2-ventricle cardiac lesions, respectively. We note that multiple factors may 

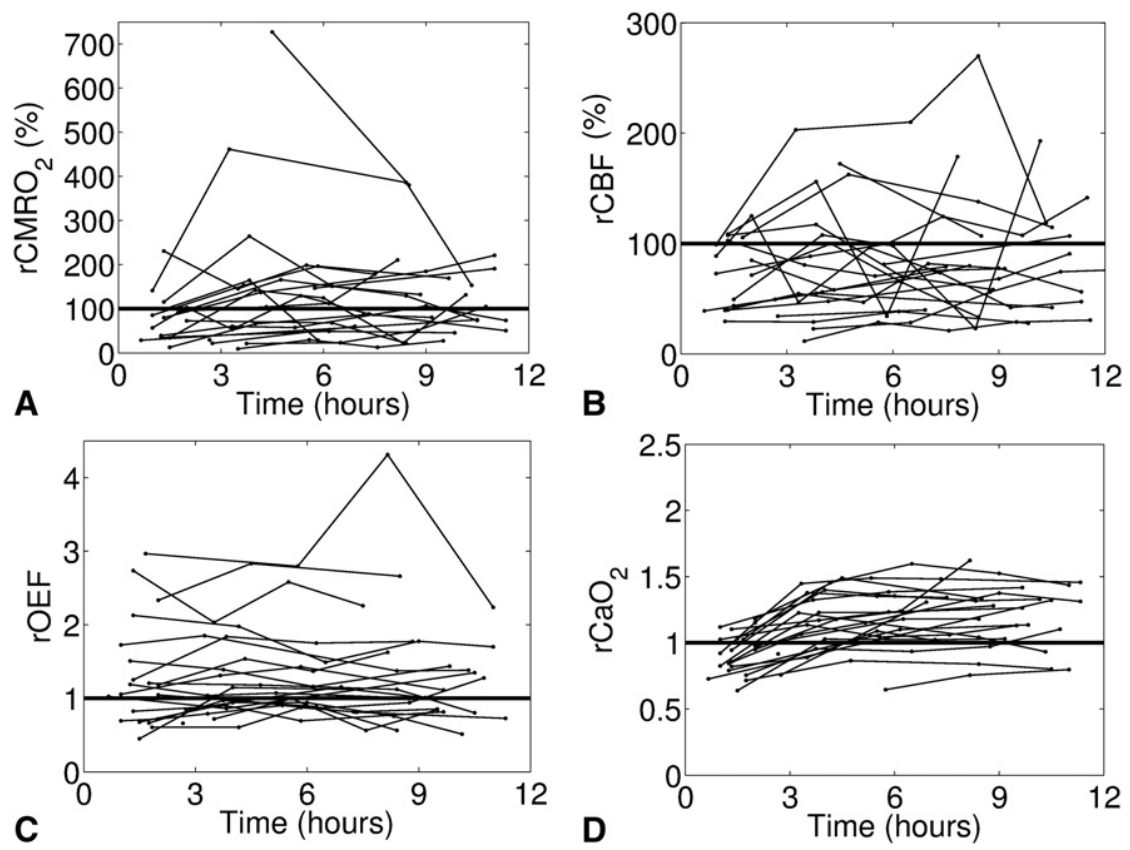

FIGURE 1. Time profiles of the relative change from preoperative levels in each outcome variable $-\mathrm{rCMRO}_{2}(\mathrm{~A}), \mathrm{rCBF}(\mathrm{B}), \mathrm{rOEF}(\mathrm{C})$, and $\mathrm{rCaO} \mathrm{O}_{2}(\mathrm{D})$ after removal from CPB for all patients with single-ventricle lesions. The horizontal line indicates no change from preoperative levels. The $x$-axis (time) is standardized between patients by setting $t=0$ as the time the patient was taken off CPB. $r \mathrm{CMRO}_{2}$, Relative change in postoperative cerebral metabolic rate of oxygen; $r C B F$, relative change in postoperative cerebral blood flow; $r O E F$, relative change in postoperative oxygen extraction fraction; $r C a \mathrm{O}_{2}$, relative change in postoperative arterial oxygen concentration; $C P B$, cardiopulmonary bypass.

contribute to the postoperative time course of these parameters, including cardiac anatomy, cerebral autoregulation, postoperative management, and postoperative physiology. The current study suggests that, in future work, it will be possible to investigate comprehensively more factors that may explain intersubject variability in $\mathrm{rCMRO}_{2}, \mathrm{rCBF}$, and rOEF. With a larger sample size, the improved statistical power will permit critical examination of the effects of systemic hemodynamics, surgical strategies, and postoperative management.

In single-ventricle patients, we observed decreases in $\mathrm{CaO}_{2}, \mathrm{ScO}_{2}, \mathrm{rCBF}$, and $\mathrm{rCMRO}_{2}$ on return to the $\mathrm{CICU}$ (at approximately 1.5 hours post-CPB, on average) from preoperative levels. At this same time point for patients with 2 ventricles, $\mathrm{CaO}_{2}$ and rOEF were increased from preoperative levels whereas $\mathrm{CBF}$ was decreased and $\mathrm{CMRO}_{2}$ was unchanged. Previous work from our laboratory suggests that preoperative $\mathrm{CBF}$ is diminished compared with healthy neonates, particularly in patients with HLHS. ${ }^{14}$ Thus, the observed decrease in CBF at the first postoperative measurement may mean that $\mathrm{CBF}$ is deranged substantially below the physiologic levels of a healthy neonate.

Because noninvasive optical imaging can be repeated over time, changes in rOEF, rCBF, and $\mathrm{rCMRO}_{2}$ were monitored further for an additional 12 hours after surgery, and a mixed-effects model was used to assess trends with time. For patients with a single ventricle, $\mathrm{rCMRO}_{2}, \mathrm{rCBF}$, and
rOEF remained unchanged during the time period of postoperative monitoring. For patients with 2 ventricles, rCBF increased significantly with time after removal from $\mathrm{CPB}$, whereas $\mathrm{rCMRO}_{2}$ and $\mathrm{rOEF}$ remained unchanged during the time period of postoperative monitoring.

During surgery, these patients encounter various degrees of hypothermia, low or no cerebral perfusion, and exposure of the blood to synthetic surfaces of the bypass machine. These profound surgical interventions have been investigated extensively as risk factors for poor neurodevelopmental outcomes, although results from different studies have been contradictory, and clear associations have not been proved. ${ }^{2-4,6-8}$ In this study, the duration of these exposures fails to explain trends in cerebral hemodynamic and metabolic measurements after surgery. Using a mixed-effect model, we found that longitudinal postoperative changes in $\mathrm{rCMRO}_{2}$ from preoperative levels were independent of DHCA duration (group 1, single ventricle) and TST (group 2, 2 ventricles). We chose to include only DHCA duration in our model for group 1 because we found that DHCA duration was highly correlated with TST (Pearson correlation coefficient, $R=0.81 ; P<.0001)$. Including 2 highly correlated variables into a mixed-effects model can lead to colinearity. For group 1, DHCA duration was also not a statistically significant predictor of rOEF or rCBF; however, a significant effect of both time after removal from $\mathrm{CPB}$ and 

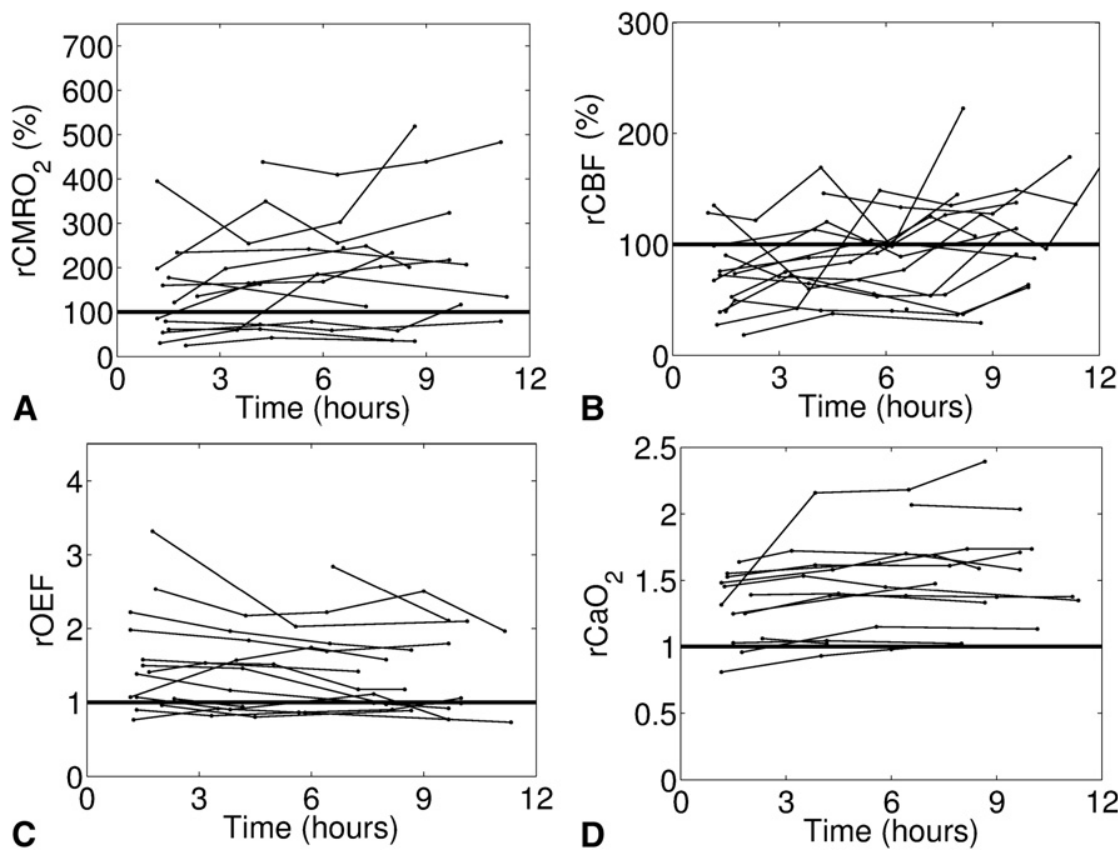

FIGURE 2. Time profiles of the relative change from preoperative levels in each outcome variable $-\mathrm{rCMRO}_{2}(\mathrm{~A}), \mathrm{rCBF}(\mathrm{B}), \mathrm{rOEF}(\mathrm{C})$, and $\mathrm{rCaO}{ }_{2}(\mathrm{D})-$ after removal from CPB for all patients with 2-ventricle lesions. The horizontal line indicates no change from preoperative levels. The $x$-axis (time) is standardized between patients by setting $t=0$ as the time the patient was taken off CPB. $r C M R O_{2}$, Relative change in postoperative cerebral metabolic rate of oxygen; $r C B F$, relative change in postoperative cerebral blood flow; $r O E F$, relative change in postoperative oxygen extraction fraction; $r C a \mathrm{O}_{2}$, relative change in postoperative arterial oxygen concentration; $C P B$, cardiopulmonary bypass.

DHCA duration was observed on $\mathrm{rCaO}_{2}$, which may reflect changes in pulmonary physiology that are extrinsic to cerebral physiology. For group 2, TST was not a significant predictor of $\mathrm{rCBF}$ or $\mathrm{rCaO}_{2}$; however, rOEF increased significantly with increasing TST.

To our knowledge, the literature investigating $\mathrm{CMRO}_{2}$ and $\mathrm{CBF}$ after $\mathrm{CPB}$ surgery in human neonates with CHD comes from a single research group. Greeley and colleagues ${ }^{10-13}$ investigated the immediate effects of cooling and circulatory arrest on $\mathrm{CMRO}_{2}$ in patients with $\mathrm{CHD}$ ranging in age from 1 day to 14 years old using arterial and jugular blood samples combined with ${ }^{133} \mathrm{Xe}$ clearance techniques for $\mathrm{CBF}$ measurements. They quantified $\mathrm{CBF}$ and $\mathrm{CMRO}_{2}$ in the operating room before $\mathrm{CPB}$, and again after rewarming and removal from $\mathrm{CPB}$. Although many differences exist between the 2 studies, the most significant differences are timing of the measurements and the average age of the cohorts. The average age of patients in the moderate hypothermia group of Greeley and colleagues ${ }^{10-13}$ was 65 months compared with 3 days in group 2 of our cohort. Despite these substantial differences in cohorts and measurement timing, patients in their studies who received DHCA (similar to group 1 in our cohort) had, on average, significantly lower $\mathrm{CMRO}_{2}$ levels after surgery compared with preoperative levels; by contrast, patients who received continuous-flow $\mathrm{CPB}$ with moderate hypothermia (similar to group 2 in our cohort) had postoperative $\mathrm{CMRO}_{2}$ that, on average, returned to preoperative levels. ${ }^{12}$ These population-averaged $\mathrm{CMRO}_{2}$ results are very similar to the results observed herein.

The majority of literature on postoperative cerebral monitoring in these patients has centered on the use of either near-infrared spectroscopy to monitor trends in regional $\mathrm{ScO}_{2}{ }^{2,20}$ or the use of mixed venous oxygen saturations from the superior vena cava. ${ }^{9,21,22}$ Our results of $\mathrm{ScO}_{2}$ both before surgery and on return to the CICU for patients with 1 ventricle (Table 3 ) are lower than previously reported, ${ }^{2,20}$ potentially because of differences in the optical measurement techniques.

\section{Study Limitations}

The results presented herein have several limitations. The most important limitation is the lack of a direct comparison group to determine the effects of DHCA versus no DHCA. Ideally, comparison should be performed on patients with 1 ventricle, half of whom have surgery with the use of DHCA and half of whom have continuous regional cerebral perfusion. This comparison would necessitate a multicenter study because surgeons in individual centers are highly biased to surgical technique.

Furthermore, the postoperative changes in $\mathrm{rCMRO}_{2}$, rOEF, and $\mathrm{rCBF}$ presented herein are indicative only of the superficial frontal cortex, as the optical techniques 
TABLE 4. Results of the mixed-effect model using time after removal from cardiopulmonary bypass and deep hypothermic circulatory arrest duration (for single-ventricle patients) or total support time (for 2-ventricle patients) as predictors for outcome measurements

\begin{tabular}{|c|c|c|c|c|}
\hline CHD type & $\begin{array}{c}\text { Outcome } \\
\text { variable }\end{array}$ & $\begin{array}{c}\text { Model } \\
\text { predictor }\end{array}$ & $\begin{array}{c}\text { Estimate }(\mathrm{SE}) \\
\text { of coefficient }\end{array}$ & $\begin{array}{c}P \\
\text { value }\end{array}$ \\
\hline \multirow[t]{8}{*}{ One ventricle } & \multirow[t]{2}{*}{$\mathrm{rCMRO}_{2}$} & Time, $\mathrm{h}$ & $0.022(0.025)$ & .42 \\
\hline & & DHCA, min & $0.007(0.009)$ & .45 \\
\hline & \multirow[t]{2}{*}{$\mathrm{rOEF}$} & Time, $\mathrm{h}$ & $-0.003(0.015)$ & .84 \\
\hline & & DHCA, min & $-0.0002(0.005)$ & .96 \\
\hline & \multirow[t]{2}{*}{$\mathrm{rCBF}$} & Time, $\mathrm{h}$ & $0.003(0.021)$ & .87 \\
\hline & & DHCA, min & $0.001(0.007)$ & .87 \\
\hline & \multirow[t]{2}{*}{$\mathrm{rCaO}_{2}$} & Time, h & $0.031(0.006)$ & $<.0001$ \\
\hline & & DHCA, min & $-0.009(0.002)$ & .0001 \\
\hline \multirow[t]{8}{*}{ Two ventricles } & \multirow[t]{2}{*}{$\mathrm{rCMRO}_{2}$} & Time, $\mathrm{h}$ & $0.045(0.030)$ & .16 \\
\hline & & TST, min & $0.003(0.003)$ & .29 \\
\hline & \multirow[t]{2}{*}{$\mathrm{rOEF}$} & Time, $\mathrm{h}$ & $-0.014(0.014)$ & .31 \\
\hline & & $\mathrm{TST}, \min$ & $0.003(0.001)$ & .008 \\
\hline & \multirow[t]{2}{*}{$\mathrm{rCBF}$} & Time, $\mathrm{h}$ & $0.046(0.019)$ & .027 \\
\hline & & TST, min & $-0.001(0.002)$ & .47 \\
\hline & \multirow[t]{2}{*}{$\mathrm{rCaO}_{2}$} & Time, $\mathrm{h}$ & $0.011(0.009)$ & .26 \\
\hline & & $\mathrm{TST}, \min$ & $0.0003(0.0007)$ & .73 \\
\hline
\end{tabular}

Logarithm transformations were used on all outcome variables to make the data more normally distributed. Coefficient estimates are reported along with SE. CHD, Congenital heart defect; $S E$, standard error; $r \mathrm{CMRO}_{2}$, relative change in the cerebral metabolic rate of oxygen; $D H C A$, deep hypothermic circulatory arrest; $r O E F$, relative change in postoperative oxygen extraction fraction; $r C B F$, relative change in postoperative cerebral blood flow; $r \mathrm{CaO}_{2}$, relative change in postoperative arterial oxygen concentration; TST, total support time.

that we used measure regional hemodynamic properties under the optical probe. Thus, we cannot make claims on $\mathrm{CMRO}_{2}, \mathrm{OEF}$, or CBF in the rest of the brain. In addition, the optical measurements were not performed beyond 12 postoperative hours because of manpower restrictions. Future work would benefit from measurements taken over multiple locations on the head for up to 48 hours after removal from CPB.

To date, $\mathrm{rCMRO}_{2}$ as measured by DOS and DCS has not been validated against any gold standard $\mathrm{CMRO}_{2}$ techniques. However, the input variables (ie, rOEF and $\mathrm{rCBF}$ ) used to derive our computation of $\mathrm{rCMRO}_{2}$ have been well validated in both animal and human models. ${ }^{15,19}$ Nevertheless, several sources of error are inherent in the estimation of $\mathrm{rCMRO}_{2}$ and are described in detail in Culver and associates. ${ }^{23}$

Given the sample size in group $1(\mathrm{n}=21)$ and the observed effect size of DHCA on $\mathrm{rCMRO}_{2}$, the study power is $83 \%$ at a significance level of 0.05 , assuming a compound symmetric correlation structure and a Pearson correlation coefficient of 0.5 between 2 measures within the same subject. This suggests that our study is slightly underpowered to detect the effects of DHCA duration on $\mathrm{rCMRO}_{2}$. However, given the sample size of group $2(n=15)$ and the observed effect size of TST on $\mathrm{rCMRO}_{2}$, the study power is greater than $90 \%$.

\section{CONCLUSIONS}

We have quantified changes noninvasively in $\mathrm{rCMRO}_{2}$, rCBF, and rOEF for 12 hours after infant cardiac surgery using DOSs in a prospective observational pilot study. We found that, despite exposures to extremes of temperature and synthetic surfaces during surgery, neither TST nor DHCA duration was a significant predictor of postoperative changes in $\mathrm{rCMRO}_{2}$ compared with preoperative levels. This work takes a useful step by quantifying the time courses of cerebral hemodynamics after surgery, from which we may discover the origin of impaired neurocognitive outcome. Future work will investigate the utility of $\mathrm{rCMRO}_{2}$ in assessing risk factors and timing of postoperative brain injury.

We thank the CICU nurses and respiratory therapists, as well as the patients and families for their participation.

\section{References}

1. Wernovsky G, Shillingford AJ, Gaynor JW. Central nervous system outcomes in children with complex congenital heart disease. Curr Opin Cardiol. 2005;20:94-9.

2. Andropoulos DB, Hunter JV, Nelson DP, Stayer SA, Stark AR, McKenzie ED, et al. Brain immaturity is associated with brain injury before and after neonatal cardiac surgery with high-flow bypass and cerebral oxygenation monitoring. $J$ Thorac Cardiovasc Surg. 2010;139:543-56.

3. Galli KK, Zimmerman RA, Jarvik GP, Wernovsky G, Kuypers MK, Clancy RR, et al. Periventricular leukomalacia is common after neonatal cardiac surgery. $J$ Thorac Cardiovasc Surg. 2004;127:692-704.

4. Chen J, Zimmerman RA, Jarvik GP, Nord AS, Clancy RR, Wernovsky G, et al. Perioperative stroke in infants undergoing open heart operations for congenital heart disease. Ann Thorac Surg. 2009;88:823-9.

5. Gaynor JW, Gerdes M, Nord AS, Bernbaum J, Zackai E, Wernovsky G, et al. Is cardiac diagnosis a predictor of neurodevelopmental outcome after cardiac surgery in infancy? J Thorac Cardiovasc Surg. 2010;140:1230-7.

6. Hovels-Gurich HH, Bauer SB, Schnitker R, Willmes-von Hinckeldey K, Messmer BJ, Seghaye MC, Huber W. Long-term neurodevelopmental outcomes in school-aged children after neonatal arterial switch operation. J Thorac Cardiovasc Surg. 2002;124:448-58.

7. Bellinger DC, Wypij D, Rivkin MJ, DeMaso DR, Robertson RL Jr, DunbarMasterson C, et al. Adolescents with D-transposition of the great arteries corrected with the arterial switch procedure: clinical perspective. Circulation. 2011;124:1361-9.

8. Fuller S, Rajagopalan R, Jarvik GP, Gerdes M, Bernbaum J, Wernovsky G, et al. Deep hypothermic circulatory arrest does not impair neurodevelopmental outcome in school-age children after infant cardiac surgery. Ann Thorac Surg. 2010;90:1985-95.

9. Hoffman GM, Ghanayem NS, Kampine JM, Berger S, Mussatto KA, Litwin SB, Tweddell JS. Venous saturation and the anaerobic threshold in neonates after the Norwood procedure for hypoplastic left heart syndrome. Ann Thorac Surg. 2000; 70:1515-20.

10. Greeley WJ, Bracey VA, Ungerleider RM, Greibel JA, Kern FH, Boyd JL, et al. Recovery of cerebral metabolism and mitochondrial oxidation state is delayed after hypothermic circulatory arrest. Circulation. 1991;84:III400-6.

11. Greeley WJ, Meliones JN, Ungerleider RM. Effect of deep hypothermia and circulatory arrest on cerebral blood flow and metabolism. Ann Thorac Surg. 1993;56:1464-6.

12. Greeley WJ, Kern FH, Ungerleider RM, Boyd JL III, Quill T, Smith LR, et al. The effect of hypothermic cardiopulmonary bypass and total circulatory arrest of cerebral metabolism in neonates, infants, and children. J Thorac Cardiovasc Surg. 1991;101:783-94.

13. Greeley WJ, Bracey VA, Ungerleider RM, Greibel JA, Kern FH, Boyd JL, et al. Effects of cardiopulmonary bypass on cerebral blood-flow in neonates, infants, and children. Circulation. 1989;80:209-15.

14. Licht DJ, Shera DM, Clancy RR, Wernovsky G, Montenegro LM, Nicolson SC, et al. Brain maturation is delayed in infants with complex congenital heart defects. J Thorac Cardiovasc Surg. 2009;137:529-37.

15. Buckley EM, Hance D, Pawlowski T, Lynch J, Wilson FB, Mesquita RC, et al. Validation of diffuse correlation spectroscopic measurement of cerebral blood 
flow using phase-encoded velocity mapping magnetic resonance imaging. $J \mathrm{Bi}$ omed Optics. 2012;17:037007-1-8.

16. Durduran T, Choe R, Baker WB, Yodh AG. Diffuse optics for tissue monitoring and tomography. Rep Prog Phys. 2010;73:076701.

17. Boas DA, Yodh AG. Spatially varying dynamical properties of turbid media probed with diffusing temporal light correlation. J Opt Soc Am A. 1997;14: 192-215.

18. Cheung C, Culver JP, Takahashi K, Greenberg JH, Yodh AG. In vivo cerebrovascular measurement combining diffuse near-infrared absorption and correlation spectroscopies. Phys Med Biol. 2001;46:2053-65.

19. Durduran T, Zhou C, Buckley EM, Kim MN, Yu G, Choe R, et al. Optical measurement of cerebral hemodynamics and oxygen metabolism in neonates with congenital heart defects. J Biomed Optics. 2010;15:037004-1-10.

20. Phelps HM, Mahle WT, Kim D, Simsic JM, Kirshbom PM, Kanter KR, Maher KO. Postoperative cerebral oxygenation in hypoplastic left heart syndrome after the Norwood procedure. Ann Thorac Surg. 2009;87:1490-4.

21. Tweddell JS, Ghanayem NS, Mussatto KA, Mitchell ME, Lamers LJ, Musa NL, et al. Mixed venous oxygen saturation monitoring after stage 1 palliation for hypoplastic left heart syndrome. Ann Thorac Surg. 2007;84:1301-11.

22. Li J, Zhang G, McCrindle BW, Holtby H, Humpl T, Cai S, et al. Profiles of hemodynamics and oxygen transport derived by using continuous measured oxygen consumption after the Norwood procedure. J Thorac Cardiovasc Surg. 2007; 133:441-8.e3.

23. Culver JP, Durduran T, Furuya D, Cheung C, Greenberg JH, Yodh AG. Diffuse optical tomography of cerebral blood flow, oxygenation, and metabolism in rat during focal ischemia. J Cereb Blood Flow Metab. 2003;23:911-24.

24. Hueber DM, Franceschini MA, Ma HY, Xu Q, Ballesteros JR, Fantini S, et al. Noninvasive and quantitative near-infrared haemoglobin spectrometry in the piglet brain during hypoxic stress, using a frequency-domain multidistance instrument. Phys Med Biol. 2001;46:41-62.

25. Mourant JR, Freyer JP, Hielscher AH, Eick AA, Shen D, Johnson TM. Mechanisms of light scattering from biological cells relevant to noninvasive opticaltissue diagnostics. Appl Optics. 1998;37:3586-93.

\section{APPENDIX 1.}

Each optical measurement session lasted approximately 10 to 15 minutes. Cerebral hemoglobin concentrations $\left(\left[\mathrm{HbO}_{2}\right]\right.$ and $\left.[\mathrm{Hb}]\right)$ and BFI were measured sequentially over the right and left frontal cortex with DOS and DCS, for a total of 4 measurements over each hemisphere. To enable the measurement of absolute $[\mathrm{Hb}]$ and $\left[\mathrm{HbO}_{2}\right]$, DOS data were also collected on a solid phantom with known optical properties during every optical measurement session. The phantom data facilitated calculation of the coupling coefficients between optical fibers and tissue. ${ }^{24}$

Diffuse optical spectroscopy data were analyzed using the semi-infinite solution to the photon diffusion equation for a homogeneous medium. The phase and AC amplitude $(A C)$ of the detected light are used to quantify the optical properties of the tissue. Specifically, the slope of phase versus source-detector separation $(r)$ and the slope of $\ln \left(A C \times r^{2}\right)$ versus $r$ were determined and used to compute tissue absorption and scattering coefficients: $\mu_{a}(\lambda)$ and $\mu_{s}^{\prime}(\lambda)$, respectively. ${ }^{16}$ Data were discarded if these linear fits had a Pearson $R^{2}<0.975$. In the near infrared, tissue absorption in brain tissue is primarily a result of $\left[\mathrm{HbO}_{2}\right]$, [Hb], and water:

$$
\mu_{a}(\lambda)=\varepsilon_{H_{b O}}(\lambda)\left[H b O_{2}\right]+\varepsilon_{H b}(\lambda)[H b]+0.75 \mu_{a, H_{2} O}(\lambda) .
$$

Here, $\varepsilon_{\mathrm{HbO}_{2}}(\lambda)$ and $\varepsilon_{\mathrm{Hb}}(\lambda)$ are the known wavelength extinction coefficients of $\mathrm{HbO}_{2}$ and $\mathrm{Hb}$, respectively, $\mu_{a, H_{2} O}(\lambda)$ is the absorption of pure water at wavelength $\lambda$, and we have assumed that the brain tissue volume is approximately $75 \%$ water. We reconstructed average [Hb] and $\left[\mathrm{HbO}_{2}\right]$ from tissue absorption measurements at 2 wavelengths $(688 \mathrm{~nm}$ and $826 \mathrm{~nm})$ via the system of 2 equations generated by Eq. 1. From [Hb] and $\left[\mathrm{HbO}_{2}\right]$, we derived $\mathrm{ScO}_{2}: \mathrm{ScO}_{2} \equiv\left[\mathrm{HbO}_{2}\right] /\left([\mathrm{Hb}]+\left[\mathrm{HbO}_{2}\right]\right) \times 100 \%$. Cerebral tissue oxygen saturation was calculated for each of the 8 measurements taken during the measurement session. These 8 values were then averaged together to quantify a mean $\mathrm{ScO}_{2}$ at each measurement time point.

For DCS, each measured intensity autocorrelation function was fit to obtain a BFI using the semi-infinite solution to the correlation diffusion equation for a homogenous medium. ${ }^{16,17}$ The measurements of BFI from the right and left cortex were averaged together to quantify mean BFI for each measurement time point. Note that the tissue optical properties $\mu_{a}$ and $\mu_{s}{ }^{\prime}$ at the DCS wavelength $(785 \mathrm{~nm})$ are required inputs to the fit for BFI. Although we only measured these coefficients at $688 \mathrm{~nm}$ and $826 \mathrm{~nm}$, we were able to derive $\mu_{a}(785 \mathrm{~nm})$ using measured $[\mathrm{Hb}]$ and $\left[\mathrm{HbO}_{2}\right]$ along with the known $\mathrm{Hb}$ and $\mathrm{HbO}_{2}$ extinction coefficients at $785 \mathrm{~nm}$ using Eq. 1. To derive $\mu_{s}^{\prime}(785 \mathrm{~nm})$, we assumed a power-law wavelength dependence for $\mu_{s}^{\prime}(\lambda)$ (ie, $\mu_{s}^{\prime}(\lambda)=A \lambda^{-b}$ ), where $A$ and $b$ depend on the size and number of scatters. ${ }^{25}$

The data acquired from DOS and DCS-namely, $\mathrm{ScO}_{2}$ and $\mathrm{BFI}$ - were combined to give a measure of change in $\mathrm{CMRO}_{2}$ by using a steady-state compartmental model of oxygen exchange between capillaries and tissue, which has been derived and discussed in detail elsewhere. ${ }^{16,23}$ Within this model, the relative change in $\mathrm{CMRO}_{2}$ (or $\mathrm{rCMRO}_{2}$ ) at time $t$ relative to the preoperative value is

$$
r C M R O_{2}(t)=r C B F(t) \times r C a O_{2}(t) \times r O E F \times 100 \% .
$$

Here, $r C B F(t) \equiv B F I(t) / B F I_{\text {pre }}$ is the relative change in postoperative BFI measured at time $t$ from the preoperative BFI value, $\mathrm{rCaO}_{2}(t) \equiv \mathrm{CaO}_{2}(t) / \mathrm{CaO}_{2 \text { pre }}$ is $\mathrm{rCaO}_{2}$, and $r O E F \equiv\left(\mathrm{SaO}_{2}(t)-\mathrm{ScO}_{2}(t)\right) /\left(\mathrm{SaO}_{2}-\mathrm{SaO}_{2}\right)_{\text {pre }} \times \mathrm{SaO}_{2 p r e}$ $\mathrm{SaO}_{2}(t)$ is rOEF. The subscript pre is used to indicate the preoperative measurement. Note that 2 terms contribute to the final estimate of $\mathrm{rCMRO}_{2}$ : relative change in oxygen delivery to tissue $\left(r \mathrm{DO}_{2} \equiv r C B F(t) \times r \mathrm{CaO}_{2}(t)\right.$ and relative change in oxygen extraction.

\section{Discussion}

Dr Christian Pizarro (Wilmington, Del). Dr Licht and colleagues have presented an important work using a novel and noninvasive technique to monitor early postoperative changes in cerebral metabolic rate compared with preoperative levels in a cohort of neonates undergoing cardiac surgery. For this purpose they evaluated 30 newborn children who underwent either the Norwood procedure or biventricular repair during the first week of life. 
Not surprisingly, the authors observed a decrease in $\mathrm{CaO}_{2}$, arterial oxygen content, and $\mathrm{ScO}_{2}$ from preoperative values in the single-ventricle subgroup, to some extent possibly influenced by reflection of controlled pulmonary blood flow and decrease in Qp/Qs.

In addition, $\mathrm{CBF}$ and $\mathrm{CMRO}_{2}$ were decreased, which reaffirms previous observations on the delayed recovery of $\mathrm{CBF}$ after DHCA. On the other hand, patients undergoing 2-ventricle repair exhibited an appropriate increase in $\mathrm{CaO}_{2}$ and content, and a decrease in CBF from preoperative levels, whereas extraction was increased significantly, which leads me to the first question.

I noticed in your slide that patients in the 2-ventricle group were extubated early after surgery. What percentage of these patients were extubated or in a weaning mode from mechanical ventilation at the time the first measurement was taken, which I understand was about 1.5 hours after separation from bypass? It is possible that some of the difference observed could be influenced by the different patient conditions at the time measurement was taken. In addition, were there any significant differences in temperature between the single-ventricle and the 2-ventricle groups?

Dr Licht. Patients with 2-ventricle repairs were extubated soon after return to the CICU from the operating room. In about half, extubation was delayed and we were able to make the initial measurement under conditions of mechanical ventilation; in the other half, extubation delayed our making our first measurement, but I don't have those numbers separated for you right now.

In terms of temperature, they were all the same temperature on arrival. I believe the protocol is separation from bypass at $35^{\circ} \mathrm{C}$ and patients are all rewarmed to $36.5^{\circ} \mathrm{C}$ on return to the CICU.

Dr Pizarro. Second, evidence suggests that cerebral vascular autoregulatory mechanisms are dysfunctional after circulatory arrest, resulting in an increased cerebrovascular resistance and decreased CBF. Moreover, the absence of a vasodilatory response to hypoxemia leaves the cerebral circulation vulnerable to changes in perfusion pressure and oxygen content in the blood. To determine the potential influence of hemodynamics on these measurements, can you tell us a little bit about some hemodynamic measurements of these patients at the time these calculations were made-for example, arterial blood pressure during the postoperative period?

Dr Licht. Our plan is to make correlations between the optical measurements and multiple vital sign measurements during this postoperative period. We use a CNS-100 Multimodal Neuromonitor (CNS Technology, LLC, Ambler, Pa) to capture all vital signs in real time off of the CNS-100 monitor through the serial port.

The optical instruments are made continuously, so we have longitudinal measurements captured in real time that are time-locked with continuous vital sign measurements. The data are quite complex and the database is quite large. We are working closely with an engineering group to develop new ways to look at the data. So far we have looked at 2-input time series analysis between blood flow and mean arterial blood pressure, and we do note that there are considerable periods of time when autoregulation is disrupted. However, if blood pressure is held constant for a long period of time, it's hard to know whether autoregulation is actually intact or disrupted, so we have to figure out how to analyze these data. We hope to have meaningful results available soon.
Dr Pizarro. Third, the arterial oxygen content in single-ventricle patients is probably influenced by several elements, including mixed venous oxygen saturation. Although I would agree that improving the lung function is one possible explanation for the observation of an increase during the postoperative period; however, a blood sample from pulmonary veins would be required to support this assertion. Increased arterial oxygen content in these patients, therefore, could be a reflection of changes in cardiac output during the postoperative period. Do you have any measures on the status of the systemic perfusion, like a mixed venous oxygen saturation trend or lactate levels, or this is part of probably the analysis that, in theory, would come in the future?

Dr Licht. Measurements of mixed venous saturations and cardiac output are not part of the research protocol and are routinely collected clinically in all patients. However, we do have a way of separating out the venous component of our cerebral oxygen saturation measurements. And if you gate the instrument to respiratory rate for those patients that are still mechanically ventilated, you can do a Fourier transform of the optical data and find the venous saturations in the brain.

Dr Pizarro. Fourth, experimental data in animals suggests that impairment in cerebral metabolism is directly related to the duration of circulatory arrest. You have shown us that $\mathrm{CMRO}_{2}$ was not affected by the duration of circulatory arrest. Can you provide your insight about the reason for this observation?

Dr Licht. That's a more difficult question and purely speculative. I believe that the brain is fully protected during that period of deep hypothermia. We do not measure metabolic recovery during rewarming from low temperatures that occurs within the operating room. Our measurements are made after the babies are returned to the CICU, a full 1.5 hours after separation from bypass. Time of measurement is important, and it is possible that the differences in $\mathrm{CMRO}_{2}$ measurements are related to this. It's our intention to develop an optical instrument that can measure brain temperature so that we can correlate temperature with return of $\mathrm{CMRO}_{2}$.

Dr Pizarro. Noninvasive assessment of $\mathrm{CMRO}_{2}$ in real time can be a very important contribution to understanding the impact of management strategies on the cerebral circulation and its effects on neurodevelopmental outcome. I congratulate you and your colleagues on this very exciting work and look forward to learning more from your future analysis.

Dr Richard Gates (Orange, Calif). A perfusion technique question: It's clear in the hypoplast group that you were using circulatory arrest and deep hypothermia. In the transposition group, is it safe to assume these patients were at full flow-say, $32^{\circ} \mathrm{C}-\mathrm{CPB}$ ?

Dr Licht. Correct.

Dr Gates. Then, apart from the other influencing factors, such as oxygen saturation and level of anesthesia, do you believe that the technique of more normothermic bypass may be the reason-as opposed to using circulatory arrest - that you saw these more favorable cerebral measurements in the transposition group?

Dr Licht. Well, it's very hard to separate because of the differences in anatomy between the 2 groups. The babies with the transposition have a corrected heart and complete separation of the arterial and venous circulations, so I believe the differences are reflective of improved arterial saturations in this population. A better comparison would be comparing infants with HLHS 
who receive surgery with DHCA and infants with HLHS who receive surgery with deep hypothermic antegrade flow with circulatory arrest, and we are looking for collaborations for that study.

Dr Gates. Yes, I would think it would be a very good study. Our group has been performing our Norwoods using continuous normothermia selective cerebral perfusion for $>7$ or 8 years and have been very pleased with the neurologic outcomes. It would be very interesting to compare a continuous selective normothermic group versus the DHCA group versus the deep hypothermia with selective perfusion group, with regard to these cerebral issues. Thank you for an excellent presentation. 


\section{APPENDIX E1.}

Pre- and first postoperative arterial blood gas data $(\mathrm{pH}$, partial pressure of carbon dioxide $\left[\mathrm{pCO}_{2}\right]$, partial pressure of oxygen $\left[\mathrm{pO}_{2}\right]$, and hematocrit) as well as systemic vital sign parameters (heart rate [HR], mean arterial pressure [MAP], transcutaneous oxygen saturation $\left[\mathrm{SpO}_{2}\right]$, right atrial pressure, and temperature) separated by cardiac lesion are summarized in Table E1. The preoperative data were obtained immediately before surgery during the optical measurements of cerebral hemodynamics. Postoperative data were obtained during normothermia and relative hemodynamic stability after the patient returned to the cardiac intensive care unit at a median time of 1.5 hours after removal from cardiopulmonary bypass. For both types of cardiac lesion, $\mathrm{pH}$ increased significantly after surgery from preoperative values and $\mathrm{pCO}_{2}$ remained relatively constant. For patients with 2-ventricle lesions, $\mathrm{pO}_{2}$ and $\mathrm{SpO}_{2}$ increased significantly after surgery, whereas for patients with 1 ventricle $\mathrm{pO}_{2}$ and $\mathrm{SpO}_{2}$ decreased significantly on average. Postoperatively, HR increased slightly in the single-ventricle group, and MAP remained unchanged in both groups.

TABLE E1. Summary of median (interquartile range) pre- and first postoperative systemic hemodynamic variables measured

\begin{tabular}{|c|c|c|c|c|}
\hline CHD type & Variable & Preoperative & Postoperative & $P$ value \\
\hline \multirow[t]{9}{*}{ Single-ventricle } & $\mathrm{pH}$ & $7.38(7.34-7.40)$ & $7.44(7.41-7.48)$ & .014 \\
\hline & $\mathrm{pCO}_{2}, \mathrm{~mm} \mathrm{Hg}$ & $40(37-43)$ & $39(36-45)$ & .99 \\
\hline & $\mathrm{pO}_{2}, \mathrm{~mm} \mathrm{Hg}$ & $53(43-61)$ & $37(34-41)$ & $<.0001$ \\
\hline & Hct, $\%$ & $41(39-44)$ & $44(40-45)$ & .08 \\
\hline & HR, bpm & $155(138-160)$ & $153(145-165)$ & .15 \\
\hline & MAP, mm Hg & $58(52-66)$ & $52(46-56)$ & .09 \\
\hline & $\mathrm{SpO}_{2}, \%$ & $92(87-95)$ & $83(80-85)$ & .004 \\
\hline & RAP, mm Hg & - & $8(5-9)$ & - \\
\hline & Temperature, ${ }^{\circ} \mathrm{C}$ & $37.1(36.7-37.3)$ & $36.0(35.6-36.4)$ & .0003 \\
\hline \multirow[t]{9}{*}{ Two ventricles } & $\mathrm{pH}$ & $7.39(7.38-7.41)$ & $7.50(7.45-7.58)$ & .003 \\
\hline & $\mathrm{pCO}_{2}, \mathrm{~mm} \mathrm{Hg}$ & $40(36-42)$ & $40(34-45)$ & .65 \\
\hline & $\mathrm{pO}_{2}, \mathrm{~mm} \mathrm{Hg}$ & $41(35-45)$ & $106(83-285)$ & $<.0001$ \\
\hline & Hct, $\%$ & $39(36-44)$ & $41(36-45)$ & .96 \\
\hline & HR, bpm & $141(138-155)$ & $159(138-177)$ & .032 \\
\hline & MAP, $\mathrm{mm} \mathrm{Hg}$ & $68(54-70)$ & $63(61-68)$ & .99 \\
\hline & $\mathrm{SpO}_{2}, \%$ & $85(78-90)$ & $96(95-99)$ & .012 \\
\hline & $\mathrm{RAP}, \mathrm{mm} \mathrm{Hg}$ & - & $6(5-7)$ & - \\
\hline & Temperature, ${ }^{\circ} \mathrm{C}$ & $37.3(37-37.5)$ & $36.2(36.0-37.0)$ & .014 \\
\hline
\end{tabular}

A Wilcoxon matched-pair test was used for differences from before the operation to after the operation. Note, RAP was not measured preoperatively. $C H D$, Congenital heart defect; $\mathrm{pCO}_{2}$, partial pressure of carbon dioxide; $\mathrm{pO}_{2}$, partial pressure of oxygen; $H c t$, hematocrit; $H R$, heart rate; bpm, beats per minute; $M A P$, mean arterial pressure; $\mathrm{SpCO}_{2}$, transcutaneous oxygen saturation; $R A P$, right atrial pressure. 